\title{
AS INTERAÇÕES EM GRUPOS DE EXPERIMENTOS DE ADUBAÇÃO DE MILHO*
}

\author{
HUMBERTO DE CAMPOS ** \\ FREDERICO PIMENTEL GOMES **
}

\section{RESUMO}

$O$ presente trabalho teve como objetivo estudar as interações lineares, N' x P'; N' x K' e P' x K', em quatro grupos de ensaios fatoriais $3^{3}$ de adubação de milho, com N, P e K, num total de 117 experimentos com 3.159 parcelas.

Os grupos eram assim constituídos:

Grupo 1: 53 ensaios, realizados em Ribeirão Preto (SP), utilizando-se as doses $0 ; 40$ e $80 \mathrm{~kg} / \mathrm{ha}$ de $\mathrm{N}$, de $\mathrm{P}_{2} \mathrm{O}_{5}$ e de $\mathrm{K}_{2} \mathrm{O}$.

Grupo 2: 41 ensaios, realizados no Estado do Rio de Janeiro, com as doses: 0,30 e $60 \mathrm{~kg} / \mathrm{ha}$ de $\mathrm{N}$ e de $\mathrm{K}_{2} \mathrm{O}$ e $0 ; 60$ e $120 \mathrm{~kg} / \mathrm{ha}$ de $\mathrm{P}_{2} \mathrm{O}_{5}$.

Grupo 3: 10 ensaios, realizados no Triângulo Mineiro, com as doses: $0 ; 75$ e $150 \mathrm{~kg} / \mathrm{ha}$ para os três nutrientes.

Grupo 4: 13 ensaios, realizados: 7 no Triângulo Mineiro e 6 em Patos de Minas (MG), utilizando-se em ambos os subgrupos, as doses: $0 ; 60$ e $120 \mathrm{~kg} / \mathrm{ha}$ para os três nutrientes.

Foi observado que somente as interações $N^{\prime} \times P^{\prime}$, referente às doses 0 e 1 no grupo 1 , e $P^{\prime} \times K^{\prime}$, referente às doses 0 e 2 no grupo 2, foram significativas.

É possível, pois, concluir com grande generalidade, que nos ensaios fatoriais $3^{3}$ de adubação de milho, quando se consideram doses moderadas de nutrientes, as interações são de importância secundária e, na grande maioria dos casos, são de pequena monta, quando comparadas aos efeitos principais dos nutrientes.

\section{INTRODUÇÃo}

Os métodos a serem adotados no estudo econômico de ensaios de adubação dependem, em grande parte, de serem ou não importantes as interações entre nutrientes. Só muito raramente, porém, podem as interações ser estimadas adequadamente em ensaios isolados, que, em geral, não têm precisão suficiente para isso. Faz-se mister, pois, aproveitar grupos de ensaios numerosos, que permitam estimar com precisão e generalidade as interações entre os nutrientes, quase sempre presentes, mas de pouca monta.

No presente trabalho serão estudadas as interações entre os componentes lineares

* Entregue para publicação em 24/4/1975.

* Professores do Departamento de Matemática e Estatística - Escola Superior de Agricultura

"Luiz de Queiroz" - USP. 
$\mathrm{N}^{\prime}, \mathrm{P}^{\prime}$ e $\mathrm{K}$, em quatro grupos de ensaios fatoriais $3^{3}$ de adubação de milho com $\mathrm{N}, \mathrm{P}$ e K, num total de 117 experimentos, com 3.159 parcelas.

\section{MATERI AL E MÉTODOS}

\section{Material}

Os experimentos, a serem estudados do ponto de vista das interações, todos eles fatoriais $3^{3}$ de adubação de milho (Zea mays L.), com N, P e K, com uma só repetição por local, estão subdivididos em grupos como se segue:

GRUPO 1: 53 ensaios em terra roxa legítima, realizados no município de Ribeirão Preto (SP), pelo Dr. Hermano Vaz de Arruda, com milho híbrido H-6999, com as doses de $0 ; 40$ e $80 \mathrm{~kg} / \mathrm{ha}$ de $\mathrm{N}$, de $\mathrm{P}_{2} \mathrm{O}_{5}$ e de $\mathrm{K}_{2} \mathrm{O}$.

GRUPO 2: 41 ensaios em solos diversos, realizados no Estado do Rio de Janeiro, sob a responsabilidade do Engo Agro Paulo Tavares de Macedo, do Instituto de Pesquisas e Experimentação Agropecuárias do Centro-Sul, com milhos híbridos (Minas 2, Vita e Agroceres). As doses utilizadas foram: 0; 30 e $60 \mathrm{~kg} / \mathrm{ha}$ de $\mathrm{N}$ e de $\mathrm{K}_{2} \mathrm{O}$, e $0 ; 60$ e $120 \mathrm{~kg} /$ ha de $\mathrm{P}_{2} \mathrm{O}_{5}$.

GRUPO 3: 10 ensaios em solos diversos, realizados no Triângulo Mineiro, sob a responsabilidade dos Engọs Agrọs Rui Alves Araujo, Carmen Silva Pereira, José Martins de Oliveira e Edwaldo Sobral Goes, do Instituto de Experimentação e Pesquisas Agropecuárias de Minas Gerais. As doses utilizadas foram:0; 75 e $150 \mathrm{~kg} / \mathrm{ha}$ para os três nutrientes.

GRUPO 4: Com dois subgrupos: um de sete ensaios realizados também no Triângulo Mineiro, sob a responsabilidade dos mesmos técnicos citados no Grupo 3, e outro de seis ensaios, realizados em Patos de Minas, pelo Engọ Agrọ José Martins de Oliveira. Em ambos os subgrupos, foram utilizadas as doses de $0 ; 60$ e $120 \mathrm{~kg} / \mathrm{ha}$ dos três nutrientes.

\section{Métodos} (1957).

Os métodos seguidos foram os utilizados por PIMENTEL GOMES e SALATI

Indicando-se por 0,1 e 2 as doses dos nutrientes, os contrastes que estimam as interações, com base nas doses 0 e 2 , são os seguintes para cada ensaio:

$$
\begin{aligned}
& N^{\prime} \times P^{\prime}=\frac{1}{6}[(000+001+002+220+221+222)-(200+201+202+020+021+022)] \\
& N^{\prime} \times K^{\prime}=\frac{1}{6}[(000+010+020+202+212+222)-(200+210+220+002+012+022)] \\
& P^{\prime} \times K^{\prime}=\frac{1}{6}[(000+100+200+022+122+222)-(020+120+220+002+102+202)]
\end{aligned}
$$


Tendo em vista as doses 0 e 1 , os contrastes estimadores das interações são os que se seguem, também para cada ensaio:

$$
\begin{aligned}
& N^{\prime} \times P^{\prime}=\frac{1}{6}[(000+001+002+110+111+112)-(100+101+102+010+011+012)] \\
& N^{\prime} \times K^{\prime}=\frac{1}{6}[(000+010+020+101+111+121)-(100+110+120+001+011+021)] \\
& P^{\prime} \times K^{\prime}=\frac{1}{6}[(000+100+200+011+111+211)-(010+110+210+001+101+201)]
\end{aligned}
$$

A análise de variância, para um número $\mathrm{E}$ de ensaios no grupo, está baseada no seguinte esquema:

\begin{tabular}{lc}
\hline Causa de Variação & Graus de Liberdade \\
\hline \hline $\begin{array}{l}\text { Interação } \\
\text { Resíduo }\end{array}$ & 1 \\
Total & $\mathrm{E}-1$ \\
\hline
\end{tabular}

\section{RESULTADOS OBTIDOS}

De conformidade com os contrastes mencionados, foram calculadas as interações para os diferentes grupos, com resultados que constam do Apêndice.

\begin{tabular}{|c|c|c|c|c|c|c|c|}
\hline \multirow{3}{*}{$\begin{array}{l}\text { Causa de } \\
\text { Variação }\end{array}$} & \multirow{3}{*}{ G.L. } & \multirow{2}{*}{\multicolumn{3}{|c|}{$\frac{\text { DOSES } 0 \text { e } 1}{\text { Quadrados Médios }}$}} & \multirow{2}{*}{\multicolumn{3}{|c|}{$\frac{\text { DOSES } 0 \text { e } 2}{\text { Quadrados Médios }}$}} \\
\hline & & & & & & & \\
\hline & & $N^{\prime} \times P^{\prime}$ & $\mathrm{N}^{\prime} \times \mathrm{K}^{\prime}$ & $\mathbf{P}^{\prime} \times \mathrm{K}^{\prime}$ & $\mathrm{N}^{\prime} \times \mathrm{P}^{\prime}$ & $\mathrm{N}^{\prime} \times \mathrm{K}^{\prime}$ & $\mathbf{P}^{\prime} \times \mathrm{K}^{\prime}$ \\
\hline Interação & 1 & 646.812 & 27.945 & 230.340 & 0,17 & 12.842 & 20.095 \\
\hline Resíduo & 52 & 141.054 & 185.735 & 114.739 & 130.173 & 147.327 & 131.842 \\
\hline
\end{tabular}

Com as estimativas obtidas forarn feitas as análises de variância, expostas a seguir.

GRUPO $1-53$ ensaios 
As interações médias, em $\mathrm{kg} / \mathrm{ha}$, com seus respectivos erros padrões são dadas a seguir.

\begin{tabular}{ccrr}
\hline DOSES & N' $^{\prime}$ P' $^{\prime}$ & N' $^{\prime}$ K'$^{\prime}$ & P' $^{\prime}$ K$^{\prime}$ \\
\hline \hline 0 e 1 & $-110,5^{*} \pm 51,6$ & $23,0 \pm 59,2$ & $65,9 \pm 46,5$ \\
0 e 2 & $-0,1 \pm 49,6$ & $-15,6 \pm 52,7$ & $-19,5 \pm 49,9$ \\
\hline
\end{tabular}

GRUPO 2 - 41 ensaios

\begin{tabular}{|c|c|c|c|c|c|c|c|}
\hline \multirow{3}{*}{$\begin{array}{l}\text { Causa de } \\
\text { Variação }\end{array}$} & \multirow{3}{*}{ G.L. } & \multicolumn{3}{|c|}{ DOSES 0 e 1} & \multicolumn{3}{|c|}{ DOSES 0 e 2} \\
\hline & & \multicolumn{3}{|c|}{ Quadrados Médios } & \multicolumn{3}{|c|}{ Quadrados Médios } \\
\hline & & $\mathbf{N}^{\prime} \times P^{\prime}$ & $\mathrm{N}^{\prime} \times \mathrm{K}^{\prime}$ & $\mathbf{P}^{\prime} \times \mathrm{K}^{\prime}$ & $N^{\prime} \times P^{\prime}$ & $\mathrm{N}^{\prime} \times \mathrm{K}^{\prime}$ & $\mathrm{P}^{\prime} \times \mathrm{K}^{\prime}$ \\
\hline Interação & 1 & 67.534 & 132.981 & 272.251 & 140.605 & 502.279 & 383.444 \\
\hline Resíduo & 40 & 77.807 & 92.091 & 168.566 & 107.413 & 157.016 & 83.235 \\
\hline
\end{tabular}

As interações médias, em $\mathrm{kg} / \mathrm{ha}$, com seus respectivos erros padrões foram as que se seguem.

\begin{tabular}{cccc}
\hline DOSES & N' $^{\prime}$ P' $^{\prime}$ & N' $^{\prime} \mathrm{K}^{\prime}$ & P' $^{\prime} \mathrm{K}^{\prime}$ \\
\hline \hline 0 e 1 & $40,6 \pm 43,6$ & $-57,0 \pm 47,4$ & $81,5 \pm 64,2$ \\
0 e 2 & $58,6 \pm 51,2$ & $-110,7 \pm 61,9$ & $96,7^{*} \pm 45,1$ \\
\hline
\end{tabular}

GRUPO 3 - 10 ensaios

\begin{tabular}{l|c|rrr||rrr}
\hline \multirow{2}{*}{$\begin{array}{l}\text { Causa de } \\
\text { Variação }\end{array}$} & \multirow{2}{*}{ G.L. } & \multicolumn{3}{|c||}{ DOSES 0 e 1 } & \multicolumn{3}{c}{ DOSES 0 e 2 } \\
\cline { 3 - 8 } & & \multicolumn{3}{|c||}{ Quadrados Médios } & \multicolumn{3}{c}{ Quadrados Médios } \\
\hline \hline Interação & 1 & 189.613 & 89.681 & 123.654 & 709.690 & 138.768 & 96 \\
Resíduo & 9 & 327.497 & 157.553 & 133.375 & 317.915 & 170.756 & 117.345 \\
\hline
\end{tabular}

A seguir, são dadas as interações médias, em $\mathrm{kg} / \mathrm{ha}$, com seus respectivos erros padrões.

\begin{tabular}{lcrr}
\hline DOSES & $\mathrm{N}^{\prime} \times \mathrm{P}^{\prime}$ & \multicolumn{1}{c}{$\mathrm{N}^{\prime} \times \mathrm{K}^{\prime}$} & \multicolumn{1}{c}{ P' $^{\prime} \mathrm{K}^{\prime}$} \\
\hline \hline 0 e 1 & $-137,7 \pm 181,1$ & $94,7 \pm 125,6$ & $-111,2 \pm 115,6$ \\
0 e 2 & $-266,4 \pm 178,4$ & $117,8 \pm 130,8$ & $-3,1 \pm 108,4$ \\
\hline
\end{tabular}


GRUPO 4 - 13 ensaios

\begin{tabular}{|c|c|c|c|c|c|c|c|}
\hline \multirow{3}{*}{$\begin{array}{l}\text { Causa de } \\
\text { Variação }\end{array}$} & \multirow{3}{*}{ G.L. } & \multicolumn{3}{|c|}{ DOSES 0 e 1} & \multicolumn{3}{|c|}{ DOSES 0 e 2} \\
\hline & & \multicolumn{3}{|c|}{ Quadrados Médios } & \multicolumn{3}{|c|}{ Quadrados Médios } \\
\hline & & $N^{\prime} \times P^{\prime}$ & $\mathbf{N}^{\prime} \times \mathrm{K}^{\prime}$ & $\mathbf{P}^{\prime} \times \mathrm{K}^{\prime}$ & $N^{\prime} \times P^{\prime}$ & $\mathrm{N}^{\prime} \times \mathrm{K}^{\prime}$ & $P^{\prime} \times \mathrm{K}$ : \\
\hline Interação & 1 & 7.584 & 60.521 & 152.714 & 163.072 & 159.508 & 2.955 \\
\hline Resíduo & 12 & 100.459 & 117.699 & 70.384 & 87.240 & 331.351 & 237.690 \\
\hline
\end{tabular}

As interações médias, em $\mathrm{kg} / \mathrm{ha}$, e seus respectivos erros padrões são dadas a seguir.

\begin{tabular}{cccc}
\hline DOSES & N'x P' $^{\prime}$ & N' $^{\prime} \mathrm{K}^{\prime}$ & \multicolumn{1}{c}{ P' $^{\prime} \mathrm{K}^{\prime}$} \\
\hline \hline 0 e 1 & $-24,2 \pm 87,9$ & $-68,2 \pm 95,2$ & $108,4 \pm 73,6$ \\
0 e 2 & $112,0 \pm 81,9$ & $110,8 \pm 159,7$ & $-15,1 \pm 135,2$ \\
\hline
\end{tabular}

\section{DISCUSSÃo E CONCLUSÓES}

Nos ensaios de adubação as opiniões lançadas a respeito da importância das interaçôes de nutrientes são, às vezes, muito controvertidas. Entretanto, diversos estudos realizados têm demonstrado que elas são relativamente de pouca monta nesse campo. Tal opinião foi confirmada, em particular, por pesquisa realizada por PIMENTEL GOMES e SALATI (1957), relativa à cana-de-açúcar, e pelos estudos de IGUE (1968), referentes ao feijoeiro.

A presente pesquisa examinou com profundidade, pela primeira vez no Brasil. o problema das interações em ensaios de adubação de milho. Ao todo se estudaram nada menos de 117 experimentos fatoriais $3^{3}$ com N, P e K, num total de 3.159 parcelas.

Verificou-se no presente estudo que somente em muito poucos casos se observaram valores significativos para as interações, confirmando assim os resultados de PIMENTEL GOMES e SALATI (1957) e IGUE (1968) para outras culturas.

No exame dos resultados para cada grupo observou-se:

\section{GRUPO 1:}

Neste caso os efeitos principais foram:

$$
\begin{array}{lll}
N_{1}-N_{0}=899 \mathrm{~kg} / \mathrm{ha} & P_{1}-P_{0}=201 \mathrm{~kg} / \mathrm{ha} & K_{1}-K_{0}=212 \mathrm{~kg} / \mathrm{ha} \\
N_{2}-N_{0}=1.311 \mathrm{~kg} / \mathrm{ha} & P_{2}-P_{0}=323 \mathrm{~kg} / \mathrm{ha} & K_{2}-K_{0}=234 \mathrm{~kg} / \mathrm{ha}
\end{array}
$$

A única interação significativa foi $N^{\prime} \times P^{\prime}=-110,5 \pm 51,6$, referente às doses 0 e 1 . Entretanto, observa-se que tal interação representa apenas $12,3 \%$ do efeito principal de $\mathrm{N}(+899 \mathrm{~kg} / \mathrm{ha})$, embora alcance $55,0 \%$ do efeito principal de $\mathrm{P}(+201 \mathrm{~kg} / \mathrm{ha})$. Assim, 
num desmembramento, teríamos:

Efeito de $\mathrm{N}$ na ausência de $\mathrm{P}=899 \cdot(-110,5)=1.009,5 \mathrm{~kg} / \mathrm{ha}$,

Efeito de $\mathrm{N}$ na presença de $\mathrm{P}=899+(-110,5)=788,5 \mathrm{~kg} / \mathrm{ha}$,

o que nos mostra que o $P$ teria uma ação inibidora sobre a resposta do $N$, uma vez que o rendimento referente ao $\mathrm{N}$ decresce de $221,0 \mathrm{~kg} / \mathrm{ha}$ com a presença do $\mathrm{P}$, o que representa uma queda de $21,9 \%$ na produção.

\section{GRUPO 2:}

Neste grupo, os efeitos principais foram:

$$
\begin{array}{lll}
N_{1}-N_{0}=335 \mathrm{~kg} / \mathrm{ha} & P_{1}-P_{0}=345 \mathrm{~kg} / \mathrm{ha} & K_{1}-K_{0}=67 \mathrm{~kg} / \mathrm{ha} \\
N_{2}-N_{0}=532 \mathrm{~kg} / \mathrm{ha} & P_{2}-P_{0}=344 \mathrm{~kg} / \mathrm{ha} & K_{2}-K_{0}=7 \mathrm{~kg} / \mathrm{ha}
\end{array}
$$

Observou-se que a interação $P^{\prime} \times K^{\prime}=96,7 \pm 45,1$, quando se consideram os niveis 0 e 2, foi a única significativa. Desdobrando convenientemente, obtemos os contrastes:

Efeito de $P$ na ausência de $K=344-(96,7)=247,3 \mathrm{~kg} / \mathrm{ha}$,

Efeito de $\mathrm{P}$ na presença de $\mathrm{K}=344+(96,7)=440,7 \mathrm{~kg} / \mathrm{ha}$.

Embora a dose dupla de $\mathrm{K}$ não tenha apresentado uma resposta satisfatória, ela age favoravelmente sobre o efeito do $\mathrm{P}$, aumentando a produção de $193,4 \mathrm{~kg} / \mathrm{ha}$ o que representa um acréscimo de $78,2 \%$.

Neste caso particular a interação $\mathrm{P}^{\prime} \mathrm{x}$ K' representou um papel preponderante nos efeitos da adubação, o que se explica em parte, pela baixa eficiência observada para o $\mathrm{K}$.

\section{GRUPOS 3 e 4:}

Para estes dois grupos, embora constituidos de um número razoável de ensaios, 10 e 13 respectivamente, não se constatou interação significativa, embora os efeitos principais, na maioria dos casos, tenham sido apreciáveis, ou seja:

GRUPO 3:

$$
\begin{array}{lll}
N_{1}-N_{0}=933 \mathrm{~kg} / \mathrm{ha} & P_{1}-P_{0}=761 \mathrm{~kg} / \mathrm{ha} & K_{1}-K_{0}=184 \mathrm{~kg} / \mathrm{ha} \\
N_{2}-N_{0}=1.159 \mathrm{~kg} / \mathrm{ha} & P_{2}-P_{0}=938 \mathrm{~kg} / \mathrm{ha} & K_{2}-K_{0}=222 \mathrm{~kg} / \mathrm{ha}
\end{array}
$$

\section{GRUPO 4:}

$$
\begin{array}{lll}
N_{1}-N_{0}=893 \mathrm{~kg} / \mathrm{ha} & P_{1}-P_{0}=530 \mathrm{~kg} / \mathrm{ha} & K_{1}-K_{0}=282 \mathrm{~kg} / \mathrm{ha} \\
N_{2}-N_{0}=1.424 \mathrm{~kg} / \mathrm{ha} & P_{2}-P_{0}=573 \mathrm{~kg} / \mathrm{ha} & K_{2}-K_{0}=317 \mathrm{~kg} / \mathrm{ha}
\end{array}
$$


Levando-se em consideração que os experimentos estudados no presente trabalho abrangeram uma grande parte da zona Brasileira produtora de milho (São Paulo, Rio de Janeiro e Minas Gerais) podemos realmente concluir com grande generalidade que nos experimentos fatoriais $3^{3}$ de adubação de milho as interações entre os nutrientes $(\mathrm{N}$, P e K) são de importância secundária e na grande maioria dos casos, são de pequena monta, quando comparadas aos efeitos principais. É claro, porém, que essa conclusão só é válida para doses moderadas de nutrientes, tais como as adotadas nos ensaios estudados.

É estranho, porém, o aparecimento de uma interação significativa de sinal negativo ( $\mathrm{N}^{\prime} \times \mathrm{P}^{\prime}=-110,5 \mathrm{~kg} / \mathrm{ha}$ no grupo 1 , para as doses 0 e 1), pois o razoável e o usual é o sinal positivo para interações significativas entre nutrientes distintos. Seria de esperar uma interação negativa entre nitrogênio e esterco, por exemplo, por conter azoto e esterco, mas nunca entre nitrogênio e fósforo.

Uma explicação razoável para esse fato, sugerida pelo Engọ Agrọ Armando Conagin, do Instituto Agronômico de Campinas, seria a de provir a resposta observada não do nitrogênio ou do fósforo, mas do enxofre, contido tanto no adubo nitrogenado (sulfato de amônio) como no adubo fosfatado (superfosfato simples).

\section{SUMMARY}

\section{INTERACTIONS IN GROUPS OF EXPERIMENTS OF FERTILIZATION OF CORN}

The present paper has in view the study of linear interactions N' $\times \mathrm{P}^{\prime} ; \mathrm{N}^{\prime} \times \mathrm{K}^{\prime}$ and $\mathrm{P}^{\prime} \mathrm{x} \mathrm{K}$ ' in four groups of $3^{3}$ factorial experiments of fertilization of corn, with $\mathrm{N}, \mathrm{P}$ and $\mathrm{K}$, involving 117 experiments and 3,159 plots.

The groups were:

Group 1: 53 experiments, carried out in Ribeirão Preto (SP), with the following levels of nutrients: $0 ; 40$ and $80 \mathrm{~kg} / \mathrm{ha}$ of $\mathrm{N}, \mathrm{P}_{2} \mathrm{O}_{5}$ and $\mathrm{K}_{2} \mathrm{O}$.

Group 2: 41 experiments, carried out in the State of Rio de Janeiro, with the levels: $0 ; 30$ and $60 \mathrm{~kg} / \mathrm{ha}$ of both $\mathrm{N}$ and $\mathrm{K}_{2} \mathrm{O}$, and $0 ; 60$ and $120 \mathrm{~kg} / \mathrm{ha}$ of $\mathrm{P}_{2} \mathrm{O}_{5}$.

Group 3: 10 experiments carried out in the Region of Triângulo Mineiro (MG) with the levels: $0 ; 75$ and $150 \mathrm{~kg} / \mathrm{ha}$ for the 3 nutrients.

Group 4: 13 experiments carried out: 7 of them in Triângulo Mineiro, and the other 6 in Patos de Minas (MG), using the levels: 0; 60 and $120 \mathrm{~kg} / \mathrm{ha}$ for each of the 3 nutrients.

It was observed that only the interaction $\mathrm{N}^{\prime} \times \mathrm{P}^{\prime}$ using the levels 0 and 1 in group 1 , and ' $P^{\prime} \times K^{\prime}$ using the levels 0 and 2 in group 2, were significant at the $5 \%$ level of probability.

Then, it is possible to conclude, with a wide generality, that in $3^{3}$ factorial ex- 
periments of fertilization of corn, for moderate levels of nutrients, the interactions have little importance. In most of the cases, interactions are secondary when compared with the main effects of nutrients.

\section{LITERATURA CITADA}

IGUE, T., 1968. Interações em Grupos de Experimentos de Adubação do Feijoeiro com N, P e K, Segundo o Esquema Fatorial $3 \times 3 \times 3$. Tese de M.S., Departamento de Matemática e Estatística da ESALQ.

PIMENTEL GOMES, F. \& E. SALATI, 1957. As interações num Grupo de Experimentos de Adubação da Cana-de-Açúcar. Rev. da Agricultura, 32:261-270. 
A PÊ N D ICE

Interações Estimadas (kg/ha)

GRUPO 1: 53 ensaios

\begin{tabular}{|c|c|c|c|c|c|c|}
\hline \multirow{2}{*}{$\begin{array}{l}\text { Número do } \\
\text { Experimento }\end{array}$} & \multicolumn{2}{|c|}{$N^{\prime} \times P^{\prime}$} & \multicolumn{2}{|c|}{$N^{\prime} \times K^{\prime}$} & \multicolumn{2}{|c|}{$P^{\prime} \times K^{\prime}$} \\
\hline & 0 e 1 & 0 e 2 & Oe 1 & 0 e 2 & 0 e 1 & 0 e 2 \\
\hline 1 & -233 & +29 & -83 & +217 & +700 & +233 \\
\hline 2 & +388 & +146 & -83 & +179 & +12 & +121 \\
\hline 3 & -179 & -421 & +8 & +225 & +371 & +250 \\
\hline 4 & -312 & +300 & -383 & -567 & -454 & +429 \\
\hline 5 & +338 & +758 & -58 & -288 & -108 & -50 \\
\hline 6 & -267 & +225 & +67 & +133 & +121 & +175 \\
\hline 7 & -292 & +383 & +1279 & -292 & -950 & -633 \\
\hline 8 & -300 & -486 & -248 & -32 & -258 & -460 \\
\hline 9 & +188 & +471 & +479 & -225 & -21 & +8 \\
\hline 10 & -343 & -688 & -952 & -250 & +52 & +192 \\
\hline 11 & -262 & +71 & -88 & -88 & -92 & -275 \\
\hline 12 & -79 & +58 & -284 & +430 & -295 & +116 \\
\hline 13 & -441 & -356 & -251 & +341 & -10 & -620 \\
\hline 14 & -104 & -321 & -54 & -58 & -717 & -675 \\
\hline 15 & +254 & +246 & -579 & -38 & +21 & -700 \\
\hline 16 & +83 & +404 & +146 & +346 & -292 & +458 \\
\hline 17 & -321 & +142 & +462 & -96 & +504 & +362 \\
\hline 18 & +17 & +183 & +475 & +217 & +25 & -33 \\
\hline 19 & +171 & +238 & -29 & -112 & +54 & +104 \\
\hline 20 & -62 & +142 & +12 & +283 & -229 & +88 \\
\hline 21 & -502 & +330 & +182 & -277 & +19 & +491 \\
\hline 22 & +29 & +26 & -198 & -264 & -166 & -315 \\
\hline 23 & +782 & -432 & -577 & -213 & -323 & -263 \\
\hline 24 & $+\tilde{6} 2$ & +238 & -108 & +183 & -29 & +254 \\
\hline 25 & +318 & +43 & +43 & +98 & +94 & +311 \\
\hline 26 & -4 & +17 & -4 & -167 & -29 & +421 \\
\hline 27 & -108 & +492 & +200 & +133 & +108 & -175 \\
\hline 28 & -192 & +108 & -333 & -75 & +238 & +217 \\
\hline 29 & -100 & -158 & +792 & +8 & +383 & -25 \\
\hline 30 & +633 & +233 & +533 & -308 & +683 & -583 \\
\hline 31 & +242 & -458 & -58 & +283 & -175 & -358 \\
\hline 32 & +233 & +250 & +233 & -250 & -392 & -592 \\
\hline 33 & +325 & -625 & -625 & +317 & +333 & -54 \\
\hline 34 & -325 & -767 & +942 & +1317 & +425 & +558 \\
\hline 35 & -192 & -75 & +608 & +483 & +383 & +458 \\
\hline 36 & -483 & +417 & +283 & -167 & +417 & +533 \\
\hline 37 & -367 & -333 & -417 & -975 & -25 & -67 \\
\hline 38 & -175 & -100 & -133 & -367 & +808 & +492 \\
\hline 39 & +167 & +158 & +275 & -442 & +150 & -350 \\
\hline 40 & -267 & +217 & +450 & -425 & +542 & -533 \\
\hline 41 & -658 & -367 & -225 & -375 & +350 & 0 \\
\hline 42 & -383 & -392 & -183 & +250 & +383 & -325 \\
\hline 43 & +475 & +617 & +367 & -442 & -75 & -300 \\
\hline 44 & -342 & $-5 \overline{8}$ & -558 & -100 & -258 & -125 \\
\hline 45 & -417 & -833 & +142 & +525 & -58 & -242 \\
\hline 46 & -625 & -358 & -83 & +133 & +142 & -258 \\
\hline 47 & -350 & +525 & 0 & +350 & +233 & 0 \\
\hline 48 & +592 & +50 & -442 & -942 & +383 & +300 \\
\hline 49 & -1267 & -25 & -758 & +483 & -150 & -300 \\
\hline 50 & -833 & -175 & +725 & +467 & -33 & +567 \\
\hline 51 & +8 & -100 & +192 & -200 & +158 & -267 \\
\hline 52 & -308 & -167 & +283 & +42 & +208 & +200 \\
\hline 53 & -67 & +175 & -167 & -233 & +333 & +208 \\
\hline
\end{tabular}


GRUPO 2: 41 ensaios

\begin{tabular}{|c|c|c|c|c|c|c|}
\hline \multirow{2}{*}{$\begin{array}{l}\text { Número do } \\
\text { Experimento }\end{array}$} & \multicolumn{2}{|c|}{$N^{\prime} \times P^{\prime}$} & \multicolumn{2}{|c|}{$N^{\prime} \times K^{\prime}$} & \multicolumn{2}{|c|}{$P^{\prime} \times K^{\prime}$} \\
\hline & 0 e 1 & 0 e 2 & 0 e 1 & Oe 2 & 0 e 1 & 0 e 2 \\
\hline 1 & -82 & +410 & -286 & +36 & -226 & +434 \\
\hline 2 & -67 & -28 & -39 & +166 & -78 & -556 \\
\hline 3 & +22 & +287 & +523 & +626 & +274 & +16 \\
\hline 4 & +278 & +524 & -197 & -806 & -422 & +49 \\
\hline 5 & +176 & +316 & -314 & +191 & +78 & +47 \\
\hline 6 & +98 & +73 & -343 & -260 & +40 & +54 \\
\hline 7 & +280 & -210 & -511 & -644 & +703 & +466 \\
\hline 8 & +336 & +40 & -438 & +568 & +1011 & +689 \\
\hline 9 & +321 & +356 & -637 & -856 & -148 & +157 \\
\hline 10 & +9 & -299 & -473 & -742 & +359 & -547 \\
\hline 11 & +71 & +241 & +318 & +467 & +149 & -39 \\
\hline 12 & -56 & +42 & +105 & -156 & -361 & -11 \\
\hline 13 & -347 & -322 & -91 & -334 & +257 & +137 \\
\hline 14 & -372 & +132 & +114 & +6 & -19 & +198 \\
\hline 15 & +178 & +278 & +185 & -211 & -690 & +12 \\
\hline 16 & +103 & +494 & -142 & -378 & -353 & -44 \\
\hline 17 & +167 & -267 & +133 & +466 & -750 & +16 \\
\hline 18 & -400 & -219 & -495 & -320 & +708 & +342 \\
\hline 19 & -229 & -468 & -118 & -292 & +217 & +4 \\
\hline 20 & +116 & -1095 & +35 & -697 & -889 & -49 \\
\hline 21 & +245 & +60 & +109 & +72 & +405 & -257 \\
\hline 22 & +229 & +197 & +356 & +334 & +571 & +866 \\
\hline 23 & +445 & +325 & +355 & +39 & +225 & +108 \\
\hline 24 & +211 & +56 & +150 & -561 & +278 & +411 \\
\hline 25 & -128 & -56 & -322 & -183 & +489 & -333 \\
\hline 26 & +416 & 0 & -450 & -394 & +144 & +194 \\
\hline 27 & -44 & +370 & -336 & +169 & +114 & +17 \\
\hline 28 & -206 & +272 & +44 & -6 & +294 & -36 \\
\hline 29 & +328 & +556 & -6 & +39 & +44 & -217 \\
\hline 30 & -247 & -89 & +170 & +22 & +180 & +550 \\
\hline 31 & -47 & -408 & -319 & +53 & -350 & +83 \\
\hline 32 & +522 & -192 & +481 & +70 & -170 & +161 \\
\hline 33 & -283 & +133 & -206 & -56 & -180 & -211 \\
\hline 34 & -14 & +528 & -478 & +147 & +303 & +106 \\
\hline 35 & +378 & +80 & -22 & -275 & -35 & -220 \\
\hline 36 & -231 & -40 & +360 & -44 & +11 & +158 \\
\hline 37 & -872 & +39 & -80 & -1016 & +880 & +455 \\
\hline 38 & +238 & +355 & +403 & +367 & +378 & +189 \\
\hline 39 & +98 & +262 & +110 & +303 & -118 & +214 \\
\hline 40 & -138 & +12 & +55 & -355 & -202 & +206 \\
\hline 41 & +132 & -344 & -38 & -93 & +220 & +146 \\
\hline
\end{tabular}


GRUPO 3: 10 ensaios

\begin{tabular}{ccccccc}
\hline $\begin{array}{c}\text { Número do } \\
\text { Experimento }\end{array}$ & \multicolumn{2}{c}{$N^{\prime} \times \mathrm{P}^{\prime}$} & \multicolumn{2}{c}{$\mathrm{N}^{\prime} \times \mathrm{K}^{\prime}$} & \multicolumn{2}{c}{$\mathrm{P}^{\prime} \times \mathrm{K}^{\prime}$} \\
\cline { 2 - 7 } & $0 \mathrm{e} 1$ & $0 \mathrm{e} 2$ & $0 \mathrm{e} 1$ & $0 \mathrm{e} 2$ & $0 \mathrm{e} 1$ & 0 e 2 \\
\hline \hline 1 & -532 & -289 & -29 & +336 & -460 & -132 \\
2 & -730 & -552 & +438 & +267 & -13 & -241 \\
3 & -622 & -1600 & +140 & +444 & +203 & +216 \\
4 & -292 & -242 & +940 & +222 & -692 & -267 \\
5 & +984 & -118 & -260 & +480 & +38 & +696 \\
6 & -292 & -89 & -197 & -457 & -457 & -508 \\
7 & +679 & +641 & -406 & -641 & +311 & -63 \\
8 & -502 & 0 & +260 & +622 & +171 & +260 \\
9 & -235 & -358 & +219 & -6 & -448 & +160 \\
10 & +165 & -57 & -158 & -89 & +235 & -152 \\
\hline
\end{tabular}

GRUPO 4: 13 ensaios

\begin{tabular}{ccccccc}
\hline \multirow{2}{*}{$\begin{array}{c}\text { Número do } \\
\text { Experimento }\end{array}$} & \multicolumn{2}{c}{$\mathrm{N}^{\prime} \times \mathrm{P}^{\prime}$} & \multicolumn{2}{c}{$\mathrm{N}^{\prime} \times \mathrm{K}^{\prime}$} & \multicolumn{2}{c}{$\mathrm{P}^{\prime} \times K^{\prime}$} \\
\cline { 2 - 7 } & $0 \mathrm{e} 1$ & $0 \mathrm{e} 2$ & 0 e 1 & 0 e 2 & 0e 1 & 0e 2 \\
\hline \hline 1 & -251 & +57 & -92 & -425 & -3 & -343 \\
2 & +349 & +221 & +624 & +446 & +121 & -243 \\
3 & +146 & -44 & -311 & -527 & -19 & -451 \\
4 & +309 & +130 & -355 & -550 & -213 & -327 \\
5 & +204 & +478 & -346 & +62 & +43 & -120 \\
6 & +70 & +818 & -444 & +1213 & +595 & +571 \\
7 & +212 & +187 & +66 & +344 & +334 & +711 \\
\hline 8 & -130 & +250 & +414 & +77 & -148 & -401 \\
9 & +201 & -125 & -377 & +690 & -160 & -23 \\
10 & -597 & +84 & +338 & +442 & -100 & +380 \\
11 & -505 & -171 & +28 & -23 & +264 & +898 \\
12 & -384 & -213 & -148 & -809 & +572 & -583 \\
13 & +62 & -216 & -284 & +500 & +123 & -265 \\
\hline
\end{tabular}

Tratamentos de 1 a 7: Triângulo Mineiro

Tratamentos de 8 a 13: Patos de Minas 
. 